Article

\title{
On Strongly Continuous Resolving Families of Operators for Fractional Distributed Order Equations
}

\author{
Vladimir E. Fedorov $1,2,3, * \mathbb{D}$ and Nikolay V. Filin 1,3 \\ 1 Department of Mathematical Analysis, Chelyabinsk State University, 454001 Chelyabinsk, Russia; \\ nikolay_filin@inbox.ru \\ 2 Laboratory of Functional Materials, South Ural State University, 454080 Chelyabinsk, Russia \\ 3 Department of Differential Equations, N.N. Krasovskii Institute of Mathematics and Mechanics of the Ural \\ Branch of the Russian Academy of Sciences, 620108 Yekaterinburg, Russia \\ * Correspondence: kar@csu.ru; Tel.: +7-351-7997-106
}

check for updates

Citation: Fedorov, V.E.; Filin, N.V. On Strongly Continuous Resolving Families of Operators for Fractional Distributed Order Equations. Fractal Fract. 2021, 5, 20. https://doi.org/ 10.3390 / fractalfract 5010020

Academic Editor: Maria Rosaria Lancia

Received: 9 January 2021

Accepted: 26 February 2021

Published: 2 March 2021

Publisher's Note: MDPI stays neutral with regard to jurisdictional claims in published maps and institutional affiliations.

Copyright: (c) 2021 by the authors. Licensee MDPI, Basel, Switzerland. This article is an open access article distributed under the terms and conditions of the Creative Commons Attribution (CC BY) license (https:// creativecommons.org/licenses/by/ $4.0 /)$.

\begin{abstract}
The aim of this work is to find by the methods of the Laplace transform the conditions for the existence of a strongly continuous resolving family of operators for a linear homogeneous equation in a Banach space with the distributed Gerasimov-Caputo fractional derivative and with a closed densely defined operator $A$ in the right-hand side. It is proved that the existence of a resolving family of operators for such equation implies the belonging of the operator $A$ to the class $\mathcal{C}_{W}(K, a)$, which is defined here. It is also shown that from the continuity of a resolving family of operators at $t=0$ the boundedness of $A$ follows. The existence of a resolving family is shown for $A \in \mathcal{C}_{W}(K, a)$ and for the upper limit of the integration in the distributed derivative not greater than 2 . As corollary, we obtain the existence of a unique solution for the Cauchy problem to the equation of such class. These results are used for the investigation of the initial boundary value problems unique solvability for a class of partial differential equations of the distributed order with respect to time.
\end{abstract}

Keywords: distributed fractional derivative; continual derivative; Cauchy problem; resolving family of operators; Laplace transform; partial differential equation; initial boundary value problem

MSC: 34G10; 35R11

\section{Introduction}

In the last couple of decades, a new branch of the theory of differential equations has emerged as equations with distributed fractional derivatives. A distributed derivative is an integral of a fractional derivative with respect to its order. Apparently, the first works on distributed order derivatives were those by Nakhushev [1,2] (they are called continual derivatives here) and Caputo [3] (mean derivatives). With the development of the field of applications of fractional integro-differential calculus, such equations began to arise in various problems in viscoelasticity theory [4], kinetic theory [5], modeling of ultraslow diffusion [6], and other scientific areas (see [7] and references therein). Numerical methods for solving equations with distributed fractional derivatives have been very actively investigated the last twenty years (see, e.g., [8,9]). Among the works in which the qualitative properties of distributed order differential operators and corresponding equations are investigated, in addition to the mentioned works of Nakhushev [1,2], we note the monograph and other works of Pskhu [10,11], series of articles by Atanacković, Oparnica and Pilipović ([12] and others), and works by Kochubei (e.g., [6]).

Let $\mathcal{Z}$ be a Banach space and $A: D_{A} \rightarrow \mathcal{Z}$ a linear closed densely defined operator, $m-1<b \leq m \in \mathbb{N}, \omega:[0, b] \rightarrow \mathbb{C}$. Consider the Cauchy problem

$$
z^{(k)}(0)=z_{k}, \quad k=0,1, \ldots, m-1,
$$


for the distributed order equation

$$
\int_{0}^{b} \omega(\alpha) D_{t}^{\alpha} z(t) d \alpha=A z(t), \quad t>0,
$$

with the Gerasimov-Caputo derivative $D_{t}^{\alpha}$. In $[13,14]$, this problem is researched for the case of a bounded operator $A$. The Cauchy problem and other initial value problems for Equation (2) and for an analogous equation with a linear degenerate operator at the distributed order derivative are studied in $[15,16]$ under the condition of the sectoriality of operators, with the Riemann-Liouville or the Gerasimov-Caputo distributed fractional derivatives in the equation.

Sufficient and necessary conditions of an unbounded closed densely defined operator $A$ for the existence of an analytic resolving family of operators for Equation (2) are found in [17-19]. Thus, an extension of the theorem on generators of analytic semigroups of operators to the case of distributed order equations is obtained. This allows investigating a unique solvability of problem (1) and (2). A theorem on perturbations of generators for analytic resolving family of operators for distributed order Equation (2) is proved. These results were applied to study of initial boundary value problems for some partial differential equations of a distributed order with respect to time.

In this work, we study by the methods of the Laplace transform the conditions of the existence of a strongly continuous resolving family of operators for distributed order Equation (2) in terms of the operator $A$. In the first section, properties of such families of operators are studied. Firstly, we consider some properties of certain functions arising after the acting of the Laplace transform on a distributed order derivative. Then, the definitions of a resolving family of operators for Equation (2) and a class $\mathcal{C}_{W}(K, a)$ of linear closed densely defined operators are introduced. It is proved that under the condition of the existence of a resolving family of operators for the distributed order equation the operator $A$ belongs to $\mathcal{C}_{W}(K, a)$, and the form of the Laplace transform of a resolving family is found. It is shown that in contrast to the fixed-order differential equations the primitive of a solution of (2) is not a solution of this equation. Therefore, we need to introduce the so-called $k$-resolving families of operators, which give solutions of the Cauchy problem $z^{(l)}(0)=z_{l}, l=0,1, \ldots, m-1, l \neq k, z^{(k)}(0)=z_{k}$ to Equation (2). It is proved that, if there exists a resolving family of operators $\{S(t) \in \mathcal{L}(\mathcal{Z}): t \geq 0\}$ of (2), then there exist $k$-resolving families of operators for every $k=1,2, \ldots, m-1$, which are explicitly expressed in terms of $\{S(t) \in \mathcal{L}(\mathcal{Z}): t \geq 0\}$. It is also shown that continuity of resolving family at $t=0$ implies the boundedness of the operator $A$.

In the second section, the proposed approximations for operators $S(t)$ allow proving the existence of the resolving family for Equation (2) with $A \in \mathcal{C}_{W}(K, a)$ and $b \in(0,2]$. As a corollary, we obtain the unique solvability theorem for problem (1) and (2) at $b \in(0,2]$. In the third section, the abstract results are applied to the study of initial boundary value problems for a class of partial differential equations of the distributed order with respect to time.

\section{Properties of Resolving Families of Operators}

Let $\mathcal{Z}$ be a Banach space. Denote $\overline{\mathbb{R}}_{+}:=\mathbb{R}_{+} \cup\{0\}, \overline{\mathbb{R}}_{-}:=\mathbb{R}_{-} \cup\{0\}$, for $\beta>0, t>0$, $h: \mathbb{R}_{+} \rightarrow \mathcal{Z}$

$$
J_{t}^{\beta} h(t):=\frac{1}{\Gamma(\beta)} \int_{0}^{t}(t-s)^{\beta-1} h(s) d s
$$

is the Riemann-Liouville integral. Let $m-1<\alpha \leq m \in \mathbb{N}, D_{t}^{m}$ be the usual derivative of the $m$ th order, $D_{t}^{\alpha}$ be the Gerasimov-Caputo derivative of $h: \mathbb{R}_{+} \rightarrow \mathcal{Z}$ [20-22]: 


$$
D_{t}^{\alpha} h(t):=D_{t}^{m} J_{t}^{m-\alpha}\left(h(t)-\sum_{k=0}^{m-1} h^{(k)}(0) \frac{t^{k}}{k !}\right) .
$$

By $\operatorname{Lap}(\mathcal{Z})$, denote the set of functions $h: \mathbb{R}_{+} \rightarrow \mathcal{Z}$, such that the Laplace transform, which will be denoted by $\widehat{h}$ or Lap $[h]$, is defined. The Laplace transform of the GerasimovCaputo derivative of the order $\alpha>0$ satisfies the equality (see, e.g., [23])

$$
\widehat{D_{t}^{\alpha} h}(\lambda)=\lambda^{\alpha} \widehat{h}(\lambda)-\sum_{k=0}^{m-1} h^{(k)}(0) \lambda^{\alpha-1-k}
$$

Denote by $\mathcal{L}(\mathcal{Z})$ the Banach space of all linear continuous operators from $\mathcal{Z}$ to $\mathcal{Z}$, and by $\mathcal{C l}(\mathcal{Z})$ denote the set of all linear closed operators, densely defined in $\mathcal{Z}$, acting in the space $\mathcal{Z}$. We supply the domain $D_{A}$ of an operator $A \in \mathcal{C l}(\mathcal{Z})$ by the norm of its graph and, thus, we get the Banach space $D_{A}$.

Consider the Cauchy problem

$$
z(0)=z_{0}, \quad z^{(k)}(0)=0, \quad k=1,2, \ldots, m-1,
$$

for a distributed order equation

$$
\int_{0}^{b} \omega(\alpha) D_{t}^{\alpha} z(t) d \alpha=A z(t), \quad t>0,
$$

where $0 \leq m-1<b \leq m \in \mathbb{N}, \omega:[0, b] \rightarrow \mathbb{C}, A \in \mathcal{C l}(\mathcal{Z})$. By a solution of problem (4), (5) we call such function $z \in C^{m-1}\left(\overline{\mathbb{R}}_{+} ; \mathcal{Z}\right) \cap C\left(\mathbb{R}_{+} ; D_{A}\right)$ that there exists $\int_{0}^{b} \omega(\alpha) D_{t}^{\alpha} z(t) d \alpha \in$ $C\left(\mathbb{R}_{+} ; \mathcal{Z}\right)$ and equalities (4) and (5) are fulfilled.

$$
\text { Denote } W_{k}(\lambda):=\int_{k}^{b} \omega(\alpha) \lambda^{\alpha} d \alpha, k=0,1, \ldots, m-1, W(\lambda):=W_{0}(\lambda) .
$$

Lemma 1. Let $b>0, \omega \in L_{1}(0, b ; \mathbb{C})$. Then, $W_{k}, k=0,1, \ldots, m-1$, are analytic on the set $\mathbb{C} \backslash\{\lambda \in \mathbb{C}: \operatorname{Re} \lambda \leq 0\}$.

Proof. This assertion is proved for $W_{0}$ in [17]; for other $W_{k}$, it can be proved similarly.

Lemma 2. Let $b>0, \omega:(0, b) \rightarrow \mathbb{C}$ be a piecewise continuous function, which be continuous from the left at the point $b, \omega(b) \neq 0$. Then, for every $k=0,1, \ldots, m-1$,

$$
\forall \varepsilon \in(0, b) \quad \exists C, \varrho>0 \quad \forall \lambda \in\{\lambda \in \mathbb{C}:|\lambda| \geq \varrho\} \backslash \overline{\mathbb{R}}_{-} \quad\left|W_{k}(\lambda)\right| \geq|\lambda|^{b-\varepsilon} / C .
$$

Proof. Since $\omega(b) \neq 0$, we have $\operatorname{Re}\left(\omega(\alpha) e^{i \alpha \arg \lambda}\right) \neq 0$ for all $\alpha \in(c, b)$, where $c \in(0, b)$ is close enough to $b$. Hence, we have for some $C_{1}=C_{1}\left(\varepsilon_{1}\right)>0$ and for all large enough $|\lambda|$

$$
\begin{aligned}
& \left|\int_{c}^{b} \omega(\alpha) \lambda^{\alpha} d \alpha\right| \geq\left|\operatorname{Re}\left(\int_{c}^{b} \omega(\alpha) \lambda^{\alpha} d \alpha\right)\right|=\left.\left|\int_{c}^{b} \operatorname{Re}\left(\omega(\alpha) e^{i \alpha \arg \lambda}\right)\right| \lambda\right|^{\alpha} d \alpha \mid \\
= & \left.\left.\left|\operatorname{Re}\left(\omega(\xi) e^{i \xi \arg \lambda}\right)\right|\left|\int_{c}^{b}\right| \lambda\right|^{\alpha} d \alpha|=| \operatorname{Re}\left(\omega(\xi) e^{i \xi \arg \lambda}\right)|| \frac{|\lambda|^{b}-|\lambda|^{c}}{\ln |\lambda|}\left|\geq C_{1}\right| \lambda\right|^{\varepsilon_{1}}
\end{aligned}
$$

for some $\xi \in(c, b)$ and every $\varepsilon_{1} \in(c, b)$. Thus, at $\varepsilon_{2} \in\left(c, \varepsilon_{1}\right)$ for some $C_{2}>0$ and large enough $|\lambda|$ 


$$
\left|\int_{k}^{b} \omega(\alpha) \lambda^{\alpha} d \alpha\right| \geq C_{1}|\lambda|^{\varepsilon_{1}}-|\lambda|^{c} \int_{k}^{c}|\omega(\alpha)| d \alpha \geq C_{2}|\lambda|^{\varepsilon_{2}} .
$$

Take $\varepsilon=b-\varepsilon_{2}, C=C_{2}^{-1}$.

Lemma 3. Let $b>0, \omega:(0, b) \rightarrow \mathbb{C}$ be a bounded a.e. on $(0, b)$ function. Then, for all $k, l=0,1, \ldots, m-1, k>l$,

$$
\exists C, \varrho>0 \quad \forall \lambda \in\{\lambda \in \mathbb{C}:|\lambda| \geq \varrho\} \backslash \overline{\mathbb{R}}_{-} \quad\left|W_{k}(\lambda)-W_{l}(\lambda)\right| \leq C|\lambda|^{k} .
$$

Proof. We have for $\lambda \in\{\lambda \in \mathbb{C}:|\lambda| \geq \varrho\} \backslash \overline{\mathbb{R}}_{\text {- }}$ at some $\varrho>0$

$$
\left|W_{k}(\lambda)-W_{l}(\lambda)\right|=\left|\int_{l}^{k} \omega(\alpha) \lambda^{\alpha} d \alpha\right| \leq C_{1}\left|\frac{|\lambda|^{k}-|\lambda|^{l}}{\ln |\lambda|}\right| \leq C|\lambda|^{k} .
$$

A family of operators $\{S(t) \in \mathcal{L}(\mathcal{Z}): t \geq 0\}$ is called resolving for Equation (5), if the next conditions are satisfied:

(i) $S(t)$ is strongly continuous at $t \geq 0, S(0)=I$.

(ii) $S(t)\left[D_{A}\right] \subset D_{A}, S(t) A z=A S(t) z$ for all $z \in D_{A}, t \geq 0$.

(iii) $S(t) z_{0}$ is a solution of Cauchy problem (4) and (5) for all $z_{0} \in D_{A}$.

A resolving family of operators $\{S(t) \in \mathcal{L}(\mathcal{Z}): t \geq 0\}$ is called exponentially bounded, if there exist $K \geq 1, a \geq 0$, such that $\|S(t)\|_{\mathcal{L}(\mathcal{Z})} \leq K e^{a t}$ for all $t \geq 0$.

Remark 1. Concepts of the resolving family of operators are used in the study of first order equations [24-26] ( $C_{0}$-continuous semigroup of operators), integro-differential equations [27], integral evolution equations [28], and fractional differential equations [23].

Denote $\mathbb{N}_{0}:=\mathbb{N} \cup\{0\}$. An operator $A \in \mathcal{C l}(\mathcal{Z})$ is called the operator of the class $\mathcal{C}_{W}(K, a)$ for some $K \geq 1, a \geq 0$, if the following two conditions are fulfilled:

(i) If $\operatorname{Re} \lambda>a$, then $W(\lambda) \in \rho(A)$.

(ii) If $\operatorname{Re} \lambda>a$, then for all $n \in \mathbb{N}_{0}\left\|\frac{d^{n}}{d \lambda^{n}}\left(\frac{W(\lambda)}{\lambda}(W(\lambda) I-A)^{-1}\right)\right\|_{\mathcal{L}(\mathcal{Z})} \leq \frac{K n !}{(\operatorname{Re} \lambda-a)^{n+1}}$.

Here, we suppose that $\omega \in L_{1}(0, b ; \mathbb{C})$; , hence, due to Lemma 1 and the properties of an operator resolvent, there exist the derivatives in Condition (ii) at $\operatorname{Re} \lambda>a$.

Theorem 1. Let $b>0, \omega \in L_{1}(0, b ; \mathbb{C})$; there exists a resolving family of operators $\{S(t) \in$ $\mathcal{L}(\mathcal{Z}): t \geq 0\}$ for Equation (5), such that for all $t \geq 0\|S(t)\|_{\mathcal{L}(\mathcal{Z})} \leq K e^{a t}$ at some $K \geq 1, a \geq 0$. Then, $A \in \mathcal{C}_{W}(K, a)$ and there exists the Laplace transform $\widehat{S}(\lambda)=\frac{W(\lambda)}{\lambda}(W(\lambda) I-A)^{-1}$ at $\operatorname{Re} \lambda>a$.

Proof. Let there exist a resolving family of operators $\{S(t) \in \mathcal{L}(\mathcal{Z}): t \geq 0\}$ for Equation (5), for all $t \geq 0\|S(t)\|_{\mathcal{L}(\mathcal{Z})} \leq K e^{a t}$ at some $K \geq 1, a \geq 0$. Then, at $\operatorname{Re} \lambda>a$, there exists $\widehat{S}(\lambda)$. From Equation (5), due to Paragraphs (ii) and (iii) of the resolving family definition, we obtain at $z_{0} \in D_{A}$ equalities

$$
\int_{0}^{b} \omega(\alpha) D_{t}^{\alpha} S(t) z_{0} d \alpha=A S(t) z_{0}=S(t) A z_{0}
$$

hence, due to the closedness of the operator $A$ at $\operatorname{Re} \lambda>a \widehat{S}(\lambda)\left[D_{A}\right] \subset D_{A}$, and by virtue of (3) 
$\operatorname{Lap}\left[\int_{0}^{b} \omega(\alpha) D_{t}^{\alpha} S(t) z_{0} d \alpha\right](\lambda)=W(\lambda) \widehat{S}(\lambda) z_{0}-\frac{W(\lambda)}{\lambda} z_{0}=\widehat{S}(\lambda) A z_{0}=A \widehat{S}(\lambda) z_{0}$.

Therefore, the operator $W(\lambda) I-A: D_{A} \rightarrow \mathcal{Z}$ is bijective and $\widehat{S}(\lambda)=\frac{W(\lambda)}{\lambda}$ $(W(\lambda) I-A)^{-1}$ for all $\lambda \in \mathbb{C}$, such that $\operatorname{Re} \lambda>a$, and at $n \in \mathbb{N}$

$$
\frac{d^{n}}{d \lambda^{n}}\left(\frac{W(\lambda)}{\lambda}(W(\lambda) I-A)^{-1}\right)=(-1)^{n} \int_{0}^{\infty} t^{n} e^{-\lambda t} S(t) d t
$$

Therefore, Conditions (i) and (ii) of the definition of the class $\mathcal{C}_{W}(K, a)$ are fulfilled.

Remark 2. Let $b>1, z^{0}(t)$ be a solution of Cauchy problem (4) and (5) and $\left\|z^{0}(t)\right\|_{\mathcal{L}(\mathcal{Z})} \leq K e^{a t}$ at some $K \geq 1, a \geq 0$. Then, it can be shown that

$$
z^{0}(t)=\frac{1}{2 \pi i} \int_{c-i \infty}^{c+i \infty} \frac{W(\lambda)}{\lambda}(W(\lambda) I-A)^{-1} e^{\lambda t} z_{0} d \lambda:=S_{0}(t) z_{0}, \quad c>a
$$

if there exist the inverse operators $(W(\lambda) I-A)^{-1}$ and integral (8). If $z^{1}(t)$ is such solution of the Cauchy problem $z(0)=0, z^{(1)}(0)=z_{1}, z^{(k)}(0)=0, k=2,3, \ldots, m-1$, for Equation (5), then

$$
\begin{gathered}
z^{1}(t)=\frac{1}{2 \pi i} \int_{c-i \infty}^{c+i \infty} \frac{W_{1}(\lambda)}{\lambda^{2}}(W(\lambda) I-A)^{-1} e^{\lambda t} z_{1} d \lambda:=S_{1}(t) z_{1} \\
\neq J_{t}^{1} S_{0}(t) z_{1}=\frac{1}{2 \pi i} \int_{c-i \infty}^{c+i \infty} \frac{W(\lambda)}{\lambda^{2}}(W(\lambda) I-A)^{-1} e^{\lambda t} z_{1} d \lambda, \quad c>a .
\end{gathered}
$$

Thus, we see that, in contrast to linear homogeneous equations of a fixed order, $S_{1}(t) \neq$ $J_{t}^{1} S_{0}(t)$. In other words, for a solution $z(t)$ of Equation (5), $J_{t}^{1} z(t)$ is not a solution, generally speaking. Therefore, we need to consider resolving operators families $\left\{S_{k}(t): t \geq 0\right\}$, $k=0,1, \ldots, m-1$, corresponding to every initial problem

$$
z^{(l)}(0)=0, \quad l=0,1, \ldots, m-1, \quad l \neq k, \quad z^{(k)}(0)=z_{k} .
$$

A family of operators $\left\{S_{k}(t) \in \mathcal{L}(\mathcal{Z}): t \geq 0\right\}, k \in\{1,2, \ldots, m-1\}$, is called $k$-resolving for Equation (5), if the next conditions are satisfied:

(i) $S_{k}(t)$ is strongly continuous at $t \geq 0$.

(ii) $S_{k}(t)\left[D_{A}\right] \subset D_{A}, S_{k}(t) A z=A S_{k}(t) z$ for all $z \in D_{A}, t \geq 0$.

(iii) $S_{k}(t) z_{k}$ is a solution problem (5) and (9) at every $z_{k} \in D_{A}$.

Theorem 2. Let $b>1, \omega \in L_{1}(0, b ; \mathbb{C}), W$ satisfy conditions (6) and (7); there exists a resolving family of operators $\{S(t) \in \mathcal{L}(\mathcal{Z}): t \geq 0\}$ for Equation (5), such that for all $t \geq 0\|S(t)\|_{\mathcal{L}(\mathcal{Z})} \leq$ $K e^{a t}$ at some $K \geq 1, a \geq 0$. Then, there exist $k$-resolving families $\left\{S_{k}(t) \in \mathcal{L}(\mathcal{Z}): t \geq 0\right\}$, $k=1,2, \ldots, m-1$, of $(5)$.

Proof. Theorem 1 implies that $A \in \mathcal{C}_{W}(k, a)$. Consider at $k=1,2, \ldots, m-1$ the functions

$$
\frac{W_{k}(\lambda)}{\lambda^{k} W(\lambda)}=\frac{1}{\lambda^{k}}-\frac{W(\lambda)-W_{k}(\lambda)}{\lambda^{k} W(\lambda)}
$$

Due to (6) and (7) 


$$
\left|\frac{W(\lambda)-W_{k}(\lambda)}{\lambda^{k} W(\lambda)}\right| \leq \frac{C_{1}}{|\lambda|^{b-\varepsilon}} .
$$

Since we can choose such $\varepsilon>0$, that $b-\varepsilon>1$, there exists the inverse Laplace transform

$$
w_{k}(t):=\int_{c-i \infty}^{c+i \infty} e^{\lambda t} \frac{W_{k}(\lambda)}{\lambda^{k} W(\lambda)} d \lambda, \quad c>0, \quad k=1,2, \ldots, m-1 .
$$

Here, at $k=1$, we take into account that $\lambda^{-1}=\widehat{1}$. These scalar functions $w_{k}$ are continuous at $t \geq 0$, since the integrals converge uniformly with respect to $t$ on every segment. Consequently, the operator-valued functions

$$
S_{k}(t)=\int_{0}^{t} w_{k}(t-s) S(s) d s, \quad k=1,2, \ldots, m-1,
$$

are strongly continuous at $t \geq 0$ and Paragraph (ii) of the definition of $k$-resolving family of operators is valid also.

Note that the derivatives

$$
w_{k}^{(l)}(t):=\int_{c-i \infty}^{c+i \infty} e^{\lambda t} \frac{W_{k}(\lambda)}{\lambda^{k-l} W(\lambda)} d \lambda, \quad c>0, \quad l=1,2, \ldots, k-1, \quad k=1,2, \ldots, m-1
$$

are continuous at $t \geq 0$ as well, since

$$
\frac{W_{k}(\lambda)}{\lambda^{k-l} W(\lambda)}=\frac{1}{\lambda^{k-l}}-\frac{W(\lambda)-W_{k}(\lambda)}{\lambda^{k-l} W(\lambda)}, \quad\left|\frac{W(\lambda)-W_{k}(\lambda)}{\lambda^{k-l} W(\lambda)}\right| \leq \frac{C_{1}}{|\lambda|^{b-l-\varepsilon}} .
$$

Moreover, relations (10) imply that $w_{k}^{(l)}(0)=0, l=0,1, \ldots, k-2, w_{k}^{(k-1)}(0)=1$. Thus, for $k=1,2, \ldots, m-1, z \in D_{A}$,

$$
\begin{gathered}
S_{k}^{(l)}(t) z=\int_{0}^{t} w_{k}^{(l)}(t-s) S(s) z d s, \quad l=1,2, \ldots, k-1, \\
S_{k}^{(l)}(t) z=S^{(l-k)}(t) z+\int_{0}^{t} w_{k}^{(k-1)} S^{(l-k+1)}(t-s) z d s, \quad l=k, k+1, \ldots, m-1,
\end{gathered}
$$

hence, $S_{k}(t) z_{k}$ satisfies initial conditions (9).

We have at $\operatorname{Re} \lambda>a$

$$
\widehat{S}_{k}(\lambda)=\widehat{w}_{k}(\lambda) \widehat{S}(\lambda)=\frac{W_{k}(\lambda)}{\lambda^{k} W(\lambda)} \frac{W(\lambda)}{\lambda}(W(\lambda) I-A)^{-1}=\frac{W_{k}(\lambda)}{\lambda^{k+1}}(W(\lambda) I-A)^{-1} .
$$

Therefore, at $z_{k} \in D_{A}$,

$$
\begin{gathered}
\operatorname{Lap}\left[\int_{0}^{b} \omega(\alpha) D_{t}^{\alpha} S_{k}(t) z_{k} d \alpha\right](\lambda)=\frac{W(\lambda) W_{k}(\lambda)}{\lambda^{k+1}}(W(\lambda) I-A)^{-1} z_{k}-\frac{W_{k}(\lambda)}{\lambda^{k+1}} z_{k} \\
=A \frac{W_{k}(\lambda)}{\lambda^{k+1}}(W(\lambda) I-A)^{-1} z_{k}=A \widehat{S}_{k}(\lambda) z_{k}=\widehat{S}_{k}(\lambda) A z_{k} .
\end{gathered}
$$


Acting by the inverse Laplace transform, we obtain that $S_{k}(t) z_{k}$ is a solution of Equation (5).

Theorem 3. Let $b>0, \omega \in L_{1}(0, b ; \mathbb{C}), W$ satisfy condition (6); there exist an exponentially bounded resolving family of operators of Equation (5). This family is continuous in the norm of $\mathcal{L}(\mathcal{Z})$, if and only if $A \in \mathcal{L}(\mathcal{Z})$.

Proof. Take $\operatorname{Re} \lambda>a$ and obtain as in the proof of Lemma 4

$$
\frac{W(\lambda)}{\lambda}(W(\lambda) I-A)^{-1}-\frac{I}{\lambda}=\int_{0}^{\infty} e^{-\lambda t}(S(t)-I) d t
$$

Let the function $\eta(t)=\|S(t)-I\|_{\mathcal{L}(\mathcal{Z})}$ be continuous on the segment $[0,1]$ and $\eta(0)=0$. For $\varepsilon>0$, take $\delta>0$ such that $\eta(t) \leq \varepsilon$ for all $t \in[0, \delta]$, then

$$
\left\|\frac{W(\lambda)}{\lambda}(W(\lambda) I-A)^{-1}-\frac{I}{\lambda}\right\|_{\mathcal{L}(\mathcal{Z})} \leq \int_{0}^{\delta} e^{-\lambda t} \eta(t) d t+\int_{\delta}^{\infty} e^{-\lambda t} \eta(t) d t \leq \frac{\varepsilon}{\lambda}+o\left(\frac{1}{\lambda}\right)
$$

as $\operatorname{Re} \lambda \rightarrow+\infty$, since $\eta(t) \leq K e^{a t}+1$ at $t \geq 0$. Consequently, for large enough $\lambda$

$$
\left\|W(\lambda)(W(\lambda) I-A)^{-1}-I\right\|_{\mathcal{L}(\mathcal{Z}}<1,
$$

hence, the operator $W(\lambda)(W(\lambda) I-A)^{-1}$ is continuously invertible, $\left[W(\lambda)(W(\lambda) I-A)^{-1}\right]^{-1}$ $=W(\lambda)^{-1}(W(\lambda) I-A) \in \mathcal{L}(\mathcal{Z})$. Thus, $A \in \mathcal{L}(\mathcal{Z})$.

Now, let $A \in \mathcal{L}(\mathcal{Z})$. In this case, we perform the resolving family of operators $\{S(t) \in \mathcal{L}(\mathcal{Z}): t \geq 0\}$ as the inverse Laplace transform of $\frac{W(\lambda)}{\lambda}(W(\lambda) I-A)^{-1}$. Indeed, set $\Gamma_{R}=\Gamma_{1, R} \cup \Gamma_{2, R}$ is a positively oriented closed loop, where

$$
\Gamma_{1, R}=\{c+i y \in \mathbb{C}: y \in[-R, R]\}, \quad \Gamma_{2, R}=\left\{c+R e^{i \varphi} \in \mathbb{C}: \varphi \in(-\pi / 2, \pi / 2)\right\},
$$

define also the contours $\Gamma_{3, R}=\{c+i y \in \mathbb{C}: y \in(R, \infty)\}, \Gamma_{4, R}=\{c+i y \in \mathbb{C}: y \in$ $(-\infty,-R)\}$, then $\{c+i y \in \mathbb{C}: y \in \mathbb{R}\}=\Gamma_{3, R} \cup \Gamma_{4, R} \cup \Gamma_{R} \backslash \Gamma_{2, R}$. Consider for large enough $c>0$ the operators at $t \geq 0$

$$
\begin{gathered}
S(t)=\frac{1}{2 \pi i} \int_{c-i \infty}^{c+i \infty} e^{\lambda t} \frac{W(\lambda)}{\lambda}(W(\lambda)-A)^{-1} d \lambda=I+\frac{1}{2 \pi i} \int_{c-i \infty}^{c+i \infty} \sum_{n=1}^{\infty} \frac{A^{n} e^{\lambda t} d \lambda}{\lambda W(\lambda)^{n}} \\
=I+\frac{1}{2 \pi i}\left(\int_{\Gamma_{R}}-\int_{\Gamma_{2, R}}+\int_{\Gamma_{3, R}}+\int_{\Gamma_{4, R}}\right) \sum_{n=1}^{\infty} \frac{A^{n} e^{\lambda t} d \lambda}{\lambda W(\lambda)^{n}} .
\end{gathered}
$$

At $\varrho>0$ from (6) for $\lambda \in\left\{c+i k \in \mathbb{C}: c>a, c^{2}+k^{2} \geq \varrho^{2}\right\}$

$$
\left\|\frac{A^{n}}{\lambda W(\lambda)^{n}}\right\|_{\mathcal{L}(\mathcal{Z})} \leq \frac{C\|A\|_{\mathcal{L}(\mathcal{Z})}^{n}}{|\lambda|^{(b-\varepsilon) n+1}}
$$

Take small $t>0$ and $R=1 / t$, then 


$$
\begin{gathered}
\left\|Z_{0}(t)-I\right\|_{\mathcal{L}(\mathcal{Z})} \leq \frac{C e}{2 \pi} \sum_{k=1}^{3} \sum_{n=1}^{\infty} \int_{\Gamma_{k, R}} \frac{\|A\|_{\mathcal{L}(\mathcal{Z})}^{n}}{|\lambda|^{(b-\varepsilon) n+1}}|d \lambda| \leq C_{1} \sum_{n=1}^{\infty} \frac{\|A\|_{\mathcal{L}(\mathcal{Z})}^{n}}{R^{(b-\varepsilon) n}} \\
=C_{1} \sum_{n=1}^{\infty} t^{(b-\varepsilon) n}\|A\|_{\mathcal{L}(\mathcal{Z})}^{n}=\frac{C_{1} t^{b-\varepsilon}\|A\|_{\mathcal{L}(\mathcal{Z})}}{1-t^{b-\varepsilon}\|A\|_{\mathcal{L}(\mathcal{Z})}} \rightarrow 0
\end{gathered}
$$

as $t \rightarrow 0+$.

Remark 3. As shown in the proof of Theorem 3, if $A \in \mathcal{L}(\mathcal{Z})$, then resolving operators of Equation (5) has the form

$$
S(t)=\sum_{n=0}^{\infty} a_{n}(t) A^{n}
$$

where

$$
a_{0}(t) \equiv 1, \quad a_{n}(t)=\frac{1}{2 \pi i} \int_{\gamma} \frac{e^{\lambda t} d \lambda}{\lambda W(\lambda)^{n}}, n \in \mathbb{N},
$$

$\gamma=\left\{R e^{i \varphi}: \varphi \in(-\pi, \pi)\right\} \cup\left\{x e^{i \pi}, x \in(-R,-\infty)\right\} \cup\left\{x e^{-i \pi}, x \in(-\infty,-R)\right\}$ at $R>0$ large enough. For equation $D_{t}^{\alpha} z(t)=A z(t)$, we have $W(\lambda)=\lambda^{\alpha}$, and we obtain using the Hankel representation for the Euler gamma function that

$$
a_{n}(t)=\frac{1}{2 \pi i} \int_{\gamma} \frac{e^{\lambda t} d \lambda}{\lambda^{\alpha n+1}}=\frac{t^{\alpha n}}{2 \pi i} \int_{t \gamma} \frac{e^{\mu} d \mu}{\mu^{\alpha n+1}}=\frac{t^{\alpha n}}{\Gamma(\alpha n+1)}, n \in \mathbb{N} .
$$

Therefore, $S(t)=\sum_{n=0}^{\infty} \frac{t^{\alpha n} A^{n}}{\Gamma(\alpha n+1)}=E_{\alpha, 1}\left(t^{\alpha} A\right)$ is the Mittag-Leffler function.

\section{Existence of Resolving Families of Operators and the Cauchy Problem Unique Solvability}

Let $A \in \mathcal{C}_{W}(K, a)$, for brevity, denote

$$
H(\lambda):=\frac{W(\lambda)}{\lambda}(W(\lambda) I-A)^{-1}, \quad \operatorname{Re} \lambda>a,
$$

and define at $n \in \mathbb{N}, t \geq 0$

$$
\mathcal{S}_{n}(t):=e^{-n t}\left(I+\sum_{k=0}^{\infty} \frac{(-1)^{k}(n(n+a) t)^{k+1}}{k !(k+1) !} H^{(k)}(n+a)\right) .
$$

The series converges uniformly on every segment $[0, T]$ and $\left\|\mathcal{S}_{n}(t)\right\|_{\mathcal{L}(\mathcal{Z})} \leq K e^{a t}$ for all $t \geq 0$. It can be shown that $\mathcal{S}_{n}(t)$ is infinitely differentiable at $t>0$. Put for $\operatorname{Re} \lambda>a$

$$
H_{n}(\lambda):=\int_{0}^{\infty} e^{-\lambda t} \mathcal{S}_{n}(t) d t
$$

then for $s>a$

$$
\mathcal{S}_{n}(t)=\int_{s-i \infty}^{s+i \infty} e^{\lambda t} H_{n}(\lambda) d \lambda
$$

Here and further, $i$ is the imaginary unit. 
Lemma 4. Let $b>0, \omega \in L_{1}(0, b ; \mathbb{C}), W$ satisfy condition (6); $A \in \mathcal{C}_{W}(K, a), r=[1 / b]+1$ at $b \in(0,1], D_{A^{r}}$ is dense in $\mathcal{Z}$. Then, for any $z \in \mathcal{Z}$, there exists the limit $\lim _{n \rightarrow \infty} \mathcal{S}_{n}(t) z$ in $\mathcal{Z}$.

Proof. It is sufficient to prove the thesis on a dense set in $\mathcal{Z}$. For $z \in D_{A}$

$$
H(\lambda) z=\frac{z}{\lambda}+\frac{H(\lambda) A z}{W(\lambda)}
$$

so that the integral

$$
\int_{s-i \infty}^{s+i \infty} e^{\lambda t} H(\lambda) z d \lambda, \quad s>a,
$$

converges. Indeed, the first term in the right-hand side of (12) is the Laplace transform of the constant $z$ and the second term is absolutely integrable at $b>1$ due to conditions (6) and $A \in \mathcal{C}_{W}(K, a)$. If $b \in(0,1]$, then $r b>1$ and for $z \in D_{A^{r}}$

$$
H(\lambda) z=\frac{z}{\lambda}+\frac{A z}{\lambda W(\lambda)}+\cdots+\frac{A^{r-1} z}{\lambda W(\lambda)^{r-1}}+\frac{H(\lambda) A^{r} z}{W(\lambda)^{r}} .
$$

Thus, integral (13) converges, if we choose a small enough number $\varepsilon>0$ in condition (6). We have for $\operatorname{Re} \lambda>a$

$$
\begin{gathered}
H_{n}(\lambda)=\int_{0}^{\infty} e^{-(n+\lambda) t}\left(I+\sum_{k=0}^{\infty} \frac{(-1)^{k}(n(n+a) t)^{k+1}}{k !(k+1) !} H^{(k)}(n+a)\right) d t \\
=\frac{I}{n+\lambda}+\sum_{k=0}^{\infty} \frac{(-1)^{k}(n(n+a))^{k+1} H^{(k)}(n+a)}{k !(n+\lambda)^{k+2}} \\
=\frac{I}{n+\lambda}+\frac{n(n+a)}{(n+\lambda)^{2}} \sum_{k=0}^{\infty}\left(\frac{\lambda(n+a)}{n+\lambda}-(n+a)\right)^{k} \frac{H^{(k)}(n+a)}{k !}=\frac{I}{n+\lambda}+\frac{n(n+a)}{(n+\lambda)^{2}} H\left(\frac{\lambda(n+a)}{n+\lambda}\right),
\end{gathered}
$$

so that $\lim _{n \rightarrow \infty} H_{n}(\lambda)=H(\lambda)$ since $H(\lambda)$ is analytic on $\{\lambda \in \mathbb{C}: \operatorname{Re} \lambda>a\}$ due to Lemma 1 . Moreover, for $\operatorname{Re} \lambda>a, z \in D_{A}$ due to (12)

$$
H_{n}(\lambda) z-\frac{z}{\lambda}=-\frac{z}{\lambda}+\frac{z}{n+\lambda}+\frac{n}{n+\lambda}\left(\frac{z}{\lambda}+\frac{(n+a) H\left(\frac{\lambda(n+a)}{n+\lambda}\right) A z}{(n+\lambda) W\left(\frac{\lambda(n+a)}{n+\lambda}\right)}\right)=\frac{n(n+a) H\left(\frac{\lambda(n+a)}{n+\lambda}\right) A z}{(n+\lambda)^{2} W\left(\frac{\lambda(n+a)}{n+\lambda}\right)} .
$$

Therefore, for all $\operatorname{Re} \lambda>a, n \in \mathbb{N}$,

$$
\left\|H_{n}(\lambda) z-\frac{z}{\lambda}\right\|_{\mathcal{L}(\mathcal{Z})} \leq \frac{C_{1} n|n+a|\|A z\|_{\mathcal{Z}}}{|n+\lambda|^{2}\left|\frac{\lambda|n+a|}{n+\lambda}\right|^{b-\varepsilon} \operatorname{Re}\left(\frac{\lambda(n+a)}{n+\lambda}-a\right)} \leq \frac{C_{2}}{|\lambda|^{b-\varepsilon}}\left|\frac{n+\lambda}{n+a}\right|^{b-\varepsilon-2} \leq \frac{C_{3}}{|\lambda|^{2}} .
$$

We can pass to the limit as $n \rightarrow \infty$ in (11) and the thesis is proved, since $D_{A}$ (or $D_{A^{r}}$ at $b \in(0,1])$ is dense in $\mathcal{Z}$. Note that the strong convergence obtained is uniform with respect to $t$ from every segment $[0, T]$.

Put $Z_{0}(t):=s-\lim _{n \rightarrow \infty} \mathcal{S}_{n}(t)$, where $s-\lim _{n \rightarrow \infty}$ denotes the limit in the strong topology.

Remark 4. It is proven that, for $z \in D_{A}$ or $z \in D_{A^{r}}$ at $b \in(0,1]$,

$$
Z_{0}(t) z=\int_{s-i \infty}^{s+i \infty} e^{\lambda t} H(\lambda) z d \lambda, \quad s>a .
$$


Lemma 5. Let $b>0, \omega \in L_{1}(0, b ; \mathbb{C}), W$ satisfy condition (6), $A \in \mathcal{C}_{W}(K, a), r=[1 / b]+1$ at $b \in(0,1], D_{A^{r}}$ is dense in $\mathcal{Z}$. Then, for all $t \geq 0\left\|Z_{0}(t)\right\|_{\mathcal{L}(\mathcal{Z})} \leq K e^{a t}, \widehat{Z}(\lambda)=H(\lambda)$ at $\operatorname{Re} \lambda>a$.

Proof. Due to the construction $Z_{0}(t)$ we have the inequality $\left\|Z_{0}(t)\right\|_{\mathcal{L}(\mathcal{Z})} \leq K e^{a t}$ by virtue of such inequality for $\mathcal{S}_{n}(t)$ at every $n \in \mathbb{N}$. Equality (14) implies that for every $z \in D_{A}$ or $z \in D_{A^{r}}$ at $b \in(0,1] \widehat{Z}_{0}(\lambda) z=H(\lambda) z$. It remains to continue this equality on $\mathcal{Z}$ due to uniform boundedness of $\{H(\lambda) \in \mathcal{L}(\mathcal{Z}): \operatorname{Re} \lambda>a\}$, which follows from the condition $A \in \mathcal{C}_{W}(K, a)$.

Theorem 4. Let $b \in(0,2], \omega \in L_{1}(0, b ; \mathbb{C}), W$ satisfy condition $(6) ; r=[1 / b]+1$ at $b \in(0,1]$, $D_{A^{r}}$ is dense in $\mathcal{Z}$. Then, there exists a resolving family of operators $\{S(t): t \geq 0\}$ for Equation (5), such that $\|S(t)\|_{\mathcal{L}(\mathcal{Z})} \leq K e^{a t}$ for some $K \geq 1, a \geq 0$, if and only if $A \in \mathcal{C}_{W}(K, a)$.

Proof. The direct assertion of this theorem is proved in Theorem 1. Let an operator $A \in \mathcal{C}_{W}(K, a)$. From the proof of Lemma 4, it follows that the family $\left\{Z_{0}(t) \in \mathcal{L}(\mathcal{Z}): t \geq 0\right\}$ is strongly continuous, and, due to Lemma 5 , it is exponentially bounded.

The operators $\frac{d^{n}}{d \lambda^{n}}\left(\frac{W(\lambda)}{\lambda}(W(\lambda) I-A)^{-1}\right)$ can be expressed in terms of sums of $(W(\lambda) I-A)^{-1}$ powers multiplied by scalar functions, therefore, for every $z \in D_{A}$

$$
\begin{gathered}
\frac{d^{n}}{d \lambda^{n}}\left(\frac{W(\lambda)}{\lambda}(W(\lambda) I-A)^{-1}\right) z \in D_{A}, \\
A \frac{d^{n}}{d \lambda^{n}}\left(\frac{W(\lambda)}{\lambda}(W(\lambda) I-A)^{-1}\right) z=\frac{d^{n}}{d \lambda^{n}}\left(\frac{W(\lambda)}{\lambda}(W(\lambda) I-A)^{-1}\right) A z,
\end{gathered}
$$

consequently, $S_{n}(t) z \in D_{A}, A S_{n}(t) z=S_{n}(t) A z$. Hence, for $z \in D_{A} Z_{0}(t) z \in D_{A}$, $A Z_{0}(t) z=Z_{0}(t) A z$.

We have at $z \in \mathcal{Z} Z_{0}(0) z=\lim _{n \rightarrow \infty} S_{n}(0) z=z$, and for $b>1, z \in D_{A}$

$$
Z_{0}^{\prime}(0) z=\lim _{n \rightarrow \infty} S_{n}^{\prime}(0) z=\lim _{n \rightarrow \infty} n[(n+a) H(n+a)-I] z=\lim _{n \rightarrow \infty} \frac{n(n+a)}{W(n+a)} H(n+a) A z=0
$$

due to (6).

Take $z(t)=Z_{0}(t) z_{0}$ at some $z_{0} \in D_{A}$; then, $\widehat{z}(\lambda)=H(\lambda) z_{0}$ due to Lemma 5 . Substitute $z(t)$ in in the left-hand side of Equation (5) and act by the Laplace transform on the obtained expression taking into account the equalities obtained above $z(0)=z_{0}$ and at $b \in(1,2]$ $z^{\prime}(0)=0$. Therefore, we obtain $W(\lambda) \widehat{z}(\lambda)-\frac{W(\lambda)}{\lambda} z_{0}=\frac{W(\lambda)^{2}}{\lambda}(W(\lambda) I-A)^{-1} z_{0}-\frac{W(\lambda)}{\lambda} z_{0}=$ $A \frac{W(\lambda)}{\lambda}(W(\lambda) I-A)^{-1} z_{0}=A \widehat{z}(\lambda)$, hence, $z(t)$ is a solution of problem (4) ad (5). Thus, $\left\{Z_{0}(t) \in \mathcal{L}(\mathcal{Z}): t \geq 0\right\}$ is a resolving family of operators for Equation (5).

Corollary 1. Let $b \in(0,1], \omega \in L_{1}(0, b), W$ satisfy condition (6); $K \geq 1, a \geq 0, A \in \mathcal{C}_{W}(K, a)$, $r=[1 / b]+1, D_{A}^{r}$ is dense in $\mathcal{Z}$. Then, for every $z_{0} \in D_{A}$, there exists a unique in the space $\operatorname{Lap}(\mathcal{Z})$ solution of the problem $z(0)=z_{0}$ for Equation (5). The solution has the form $z(t)=Z_{0}(t) z_{0}$.

Proof. Due to the previous theorem, it remains to prove the uniqueness of the solution. If there exist two solutions $z_{1}, z_{2}$ of the problem $z(0)=z_{0}$ for Equation (5) from the space $\operatorname{Lap}(\mathcal{Z})$, then their difference $y=z_{1}-z_{2} \in \operatorname{Lap}(\mathcal{Z})$ is a solution of Equation (5) and satisfies the initial condition $y(0)=0$. Acting by the Laplace transform on both parts of equation (5) and due to the initial condition we get the equality $W(\lambda) \widehat{y}(\lambda)=A \widehat{y}(\lambda)$. Since $A \in \mathcal{C}_{W}(K, a)$, at $\operatorname{Re} \lambda>a$ we get the identity $\widehat{y}(\lambda) \equiv 0$. It means that $y \equiv 0$. Therefore, $z(t)=Z_{0}(t) z_{0}$ is a unique solution at $z_{0} \in D_{A}$ in $\operatorname{Lap}(\mathcal{Z})$. 
Corollary 2. Let $b \in(1,2], \omega \in L_{1}(0, b), W, W_{1}$ satisfy conditions (6) and (7), $K \geq 1, a \geq 0$, $A \in \mathcal{C}_{W}(K, a), z_{0}, z_{1} \in D_{A}$. Then, there exists a unique in the space $\operatorname{Lap}(\mathcal{Z})$ solution of the problem

$$
z(0)=z_{0}, \quad z^{(1)}(0)=z_{1}
$$

for Equation (5). The solution has the form $z(t)=Z_{0}(t) z_{0}+Z_{1}(t) z_{1}$, where

$$
Z_{1}(t):=\int_{0}^{t} w_{1}(t-s) Z_{0}(s) d s .
$$

Proof. Recall that the function

$$
w_{1}(t):=\int_{c-i \infty}^{c+i \infty} e^{\lambda t} \frac{W_{1}(\lambda)}{\lambda W(\lambda)} d \lambda, \quad c>0 .
$$

is defined in the proof of Theorem 2 , where it is shown that the function $Z_{1}(t) z_{1}$ is a solution of the Cauchy problem $z(0)=0, z^{(1)}(0)=z_{1}$ for Equation (5).

Reasoning as in the proof of Corollary 1, it is easy to show the uniqueness of the solution in $\operatorname{Lap}(\mathcal{Z})$.

Remark 5. If we consider problem (4) and (5) on a segment $[0, T]$, then we can continue the function $y$ on $(T, \infty)$ by a continuous bounded way and, reasoning analogously, we get the uniqueness of the solution on the segment.

\section{Application to a Class of Initial-Boundary Value Problems}

Let polynomials $P_{n}(\lambda)=\sum_{j=0}^{n} c_{j} \lambda^{j} \not \equiv 0, Q_{n}(\lambda)=\sum_{j=0}^{n} d_{j} \lambda^{j}$ be given, $c_{j}, d_{j} \in \mathbb{R}, j=$ $0,1, \ldots, n, d_{n} \neq 0$. Suppose that $\Omega \subset \mathbb{R}^{d}$ is a bounded region with a smooth boundary $\partial \Omega$, operator pencil $\Lambda, B_{1}, B_{2}, \ldots, B_{p}$ is regularly elliptic [29], where

$$
\begin{gathered}
(\Lambda u)(s)=\sum_{|q| \leq 2 p} a_{q}(s) \frac{\partial{ }^{|q|} u(s)}{\partial s_{1}^{q_{1}} \partial s_{2}^{q_{2}} \ldots \partial s_{d}^{q_{d}}}, a_{q} \in C^{\infty}(\bar{\Omega}), \\
\left(B_{l} u\right)(s)=\sum_{|q| \leq p_{l}} b_{l q}(s) \frac{\partial^{|q|} u(s)}{\partial s_{1}^{q_{1}} \partial s_{2}^{q_{2}} \ldots \partial s_{d}^{q_{d}}}, \quad b_{l q} \in C^{\infty}(\partial \Omega), l=1,2, \ldots, p,
\end{gathered}
$$

$q=\left(q_{1}, q_{2}, \ldots, q_{d}\right) \in \mathbb{N}_{0}^{d},|q|=q_{1}+\cdots+q_{d}$. Define the operator $\Lambda_{1} \in \mathcal{C} l\left(L_{2}(\Omega)\right)$ with the domain $D_{\Lambda_{1}}=H_{\left\{B_{l}\right\}}^{2 p}(\Omega)$ [29] by the equality $\Lambda_{1} u=\Lambda u$. Suppose that the operator $\Lambda_{1}$ is self-adjoint; then, the spectrum $\sigma\left(\Lambda_{1}\right)$ of the operator $\Lambda_{1}$ is real and discrete [29]. Let, moreover, $\sigma\left(\Lambda_{1}\right)$ be bounded from the right and do not contain the origin and $\left\{\varphi_{k}: k \in \mathbb{N}\right\}$ be an orthonormal in $L_{2}(\Omega)$ system of eigenfunctions of the operator $\Lambda_{1}$, numbered by nonincreasing corresponding eigenvalues $\left\{\lambda_{k}: k \in \mathbb{N}\right\}$, taking into account their multiplicities.

Consider the initial-boundary value problem

$$
\begin{gathered}
u(s, 0)=u_{0}(s), s \in \Omega, \\
B_{l} \Lambda^{k} u(s, t)=0, \quad k=0,1, \ldots, n-1, \quad l=1,2, \ldots, p, \quad(s, t) \in \partial \Omega \times(0, T), \\
\int_{0}^{b} \omega(\alpha) D_{t}^{\alpha} P_{n}(\Lambda) u(s, t) d \alpha=i Q_{n}(\Lambda) u(s, t), \quad(s, t) \in \Omega \times(0, T),
\end{gathered}
$$


where $0<b \leq 1, \omega:(0, b) \rightarrow \mathbb{R}, i$ is the imaginary unit. Set $n_{0}=\max \{j \in\{0,1, \ldots, n\}$ : $\left.c_{j} \neq 0\right\}$,

$$
\begin{gathered}
\mathcal{X}=\left\{v \in H^{2 r n_{0}}(\Omega): B_{l} \Lambda^{k} v(s)=0, k=0,1, \ldots, n_{0}-1, l=1,2, \ldots, p, x \in \partial \Omega\right\}, \\
\mathcal{Y}=L_{2}(\Omega), L=P_{n}(\Lambda), \quad M=i Q_{n}(\Lambda), \\
D_{M}=\left\{v \in H^{2 r n}(\Omega): B_{l} \Lambda^{k} v(s)=0, k=0,1, \ldots, n-1, l=1,2, \ldots, p, x \in \partial \Omega\right\} .
\end{gathered}
$$

Then, $L \in \mathcal{L}(\mathcal{X} ; \mathcal{Y}), M \in \mathcal{C l}(\mathcal{X} ; \mathcal{Y})$ (moreover, $M \in \mathcal{L}(\mathcal{X} ; \mathcal{Y})$, if $n_{0}=n$, i. e. $c_{n} \neq 0$ ). Let $P_{n}\left(\lambda_{k}\right) \neq 0$ for all $k \in \mathbb{N}$; then, there exists an inverse operator $L^{-1} \in \mathcal{L}(\mathcal{Y} ; \mathcal{X})$ and problem (16)-(18) is represented as the Cauchy problem $z(0)=z_{0}$ for Equation (5), where $\mathcal{Z}=\mathcal{X}, A=L^{-1} M \in \mathcal{C} l(\mathcal{Z}), D_{A}=D_{M}, z_{0}=u_{0}(\cdot)$. We see that $D_{A^{r}}$ is dense in $\mathcal{Z}$, where $r=[1 / b]+1$.

Lemma 6. Let $b \in(0,1], \omega \in C([0, b] ; \mathbb{R}), \omega(\alpha) \geq 0$ at $\alpha \in[0, b), \omega(b)>0$, the spectrum $\sigma\left(\Lambda_{1}\right)$ does not contain the origin and zeros of the polynomial $P_{n}(\lambda)$, and designations (19)-(21) are valid. Then, $A \in \mathcal{C}_{W}(K, a)$.

Proof. Due to Lemma 1, the function $W(\lambda):=\int_{0}^{b} \omega(\alpha) \lambda^{\alpha} d \alpha$ is analytic on $\mathbb{C} \backslash\{\lambda \in \mathbb{C}$ : $\operatorname{Re} \lambda \leq 0\}$. If $\operatorname{Re} \lambda>1$, then $W(\lambda) \in\{\operatorname{Re} \lambda \geq 1\}$, since $\omega(\alpha) \geq 0$ a.e. on $(0, b)$ and $\alpha \leq 1$. For $v \in \mathcal{X}, \operatorname{Re} \lambda>0$,

$$
\begin{gathered}
\left\|(W(\lambda) I-A)^{-1} v\right\|_{\mathcal{X}}^{2}=\left\|\left(W(\lambda) I-i P_{n}(\Lambda)^{-1} Q_{n}(\Lambda)\right)^{-1}\right\|_{\mathcal{X}}^{2}=\sum_{k=1}^{\infty} \frac{\left(1+\lambda_{k}^{2 n_{0}}\right)\left|\left\langle v, \varphi_{k}\right\rangle\right|^{2}}{\left|W(\lambda)-i \frac{Q_{n}\left(\lambda_{k}\right)}{P_{n}\left(\lambda_{k}\right)}\right|^{2}} \leq \\
\leq \sum_{k=1}^{\infty} \frac{\left(1+\lambda_{k}^{2 n_{0}}\right)\left|\left\langle v, \varphi_{k}\right\rangle\right|^{2}}{|W(\lambda)|^{2}\left|1-i W(\lambda)^{-1} \frac{Q_{n}\left(\lambda_{k}\right)}{P_{n}\left(\lambda_{k}\right)}\right|^{2}} \leq \frac{\sum_{k=1}^{\infty}\left(1+\lambda_{k}^{2 n_{0}}\right)\left|\left\langle v, \varphi_{k}\right\rangle\right|^{2}}{|W(\lambda)|^{2} \min _{k \in \mathbb{N}, \operatorname{Re} \lambda>1}\left|1-i W(\lambda)^{-1} \frac{Q_{n}\left(\lambda_{k}\right)}{P_{n}\left(\lambda_{k}\right)}\right|^{2}} \leq \frac{\|v\|_{\mathcal{X}}^{2}}{|W(\lambda)|^{2}} .
\end{gathered}
$$

Here, the evident inclusion $i W(\lambda)^{-1} \frac{Q_{n}\left(\lambda_{k}\right)}{P_{n}\left(\lambda_{k}\right)} \in\{\operatorname{Im} \lambda \geq 1\} \cup\{\operatorname{Im} \lambda \leq-1\}$ is used. Thus, we have the inequality $\|\lambda H(\lambda)\|_{\mathcal{L}(\mathcal{Z})} \leq 1$.

Furthermore,

$$
\begin{gathered}
H^{\prime}(\lambda)=-\frac{W(\lambda)}{\lambda^{2}}(W(\lambda) I-A)^{-1}+\frac{W^{\prime}(\lambda)}{\lambda}(W(\lambda) I-A)^{-1}-\frac{W(\lambda) W^{\prime}(\lambda)}{\lambda}(W(\lambda) I-A)^{-2}, \\
\left\|\lambda^{2} H^{\prime}(\lambda)\right\|_{\mathcal{L}(\mathcal{Z}) \leq}\left\|W(\lambda)(W(\lambda) I-A)^{-1}\right\|_{\mathcal{L}(\mathcal{Z})}+\left\|\lambda W^{\prime}(\lambda)(W(\lambda) I-A)^{-1}\right\|_{\mathcal{L}(\mathcal{Z})^{+}} \\
+\left\|\lambda W(\lambda) W^{\prime}(\lambda)(W(\lambda) I-A)^{-2}\right\|_{\mathcal{L}(\mathcal{Z}) \leq 3,}
\end{gathered}
$$

since we can repeat the first part of the proof with $\lambda W^{\prime}(\lambda)$ instead of $W(\lambda)$. Consequently, by the result of Prüss (Theorem 0.4 at $k=0$ [28] and Corollary 2.5.2 [30]) we have $A \in \mathcal{C}_{W}(K, 1)$.

Lemma 6 and Corollary 1 imply the next unique solvability theorem.

Theorem 5. Let $b \in(0,1], \omega \in C([0, b] ; \mathbb{R}), \omega(\alpha) \geq 0$ at $\alpha \in[0, b), \omega(b)>0$, the spectrum $\sigma\left(\Lambda_{1}\right)$ do not contain the origin and zeros of the polynomial $P_{n}(\lambda), u_{0} \in D_{M}$. Then, there exists a unique solution of problem (16)-(18).

Remark 6. From the proof of Lemma 6, it follows that we can obtain the analogous result for a case $b \in(1,2]$, if we provide the positivity of the value $\min _{k \in \mathbb{N}, \operatorname{Re} \lambda>a}\left|1-i W(\lambda)^{-1} \frac{Q_{n}\left(\lambda_{k}\right)}{P_{n}\left(\lambda_{k}\right)}\right|$ at some $a>0$. 
Example 1. Take $P_{1}(\lambda) \equiv 1, Q_{1}(\lambda)=\lambda, A u=\Delta u, p=1, B_{1}=I$. Then, (16)-(18) is the initial-boundary value problem for the modified equation of the ultraslow diffusion [6], which here can be interpreted as equation of ultraslow dynamics of non-relativistic systems:

$$
\begin{gathered}
\int_{0}^{b} \omega(\alpha) D_{t}^{\alpha} u(s, t) d \alpha=i \Delta u(s, t), \quad(s, t) \in \Omega \times(0, T), \\
u(s, t)=0, \quad(s, t) \in \partial \Omega \times(0, T), \\
u(s, 0)=u_{0}(s), \quad s \in \Omega .
\end{gathered}
$$

\section{Conclusions}

In the present work, it is proved that a sufficient (for $b \in(0,2]$ ) and necessary (for a general case) condition of the existence of a strongly continuous resolving family of operators for differential equation of a distributed order (2) is $A \in \mathcal{C}_{W}(K, a)$. The approximation of such family of operators is proposed. It is proved that, if there exists a resolving family of operators $\{S(t) \in \mathcal{L}(\mathcal{Z}): t \geq 0\}$ of (2), then there exist the so-called $k$-resolving families of operators for every $k=1,2, \ldots, m-1$, which give solutions of Cauchy problem $z^{(l)}(0)=z_{l}, l=0,1, \ldots, m-1, l \neq k, z^{(k)}(0)=z_{k}$ to Equation (2). They are explicitly expressed in terms of $\{S(t) \in \mathcal{L}(\mathcal{Z}): t \geq 0\}$ by a non-trivial way, not in the form of the primitive as in the case of fixed-order differential equations. Further these results can be used for consideration of the case $b>2$ and the study of the unique solvability of the Cauchy problem for the corresponding inhomogeneous equation.

Author Contributions: Conceptualization, V.E.F.; methodology, V.E.F.; software, N.V.F.; validation, V.E.F.; formal analysis, N.V.F; investigation, V.E.F. and N.V.F.; resources, N.V.F.; data curation, N.V.F.; writing-original draft preparation, V.E.F.; writing — review and editing, V.E.F.; visualization, N.V.F.; supervision, V.E.F.; project administration, V.E.F.; and funding acquisition, V.E.F. All authors have read and agreed to the published version of the manuscript.

Funding: The reported study was funded by the Russian Foundation for Basic Research, project number 21-51-54003; and by Act 211 of Government of the Russian Federation, contract 02.A03.21.0011. It was also performed in the framework of Ural Mathematical Center research.

Data Availability Statement: Not applicable.

Conflicts of Interest: The authors declare no conflict of interest. The funders had no role in the design of the study; in the collection, analyses, or interpretation of data; in the writing of the manuscript, or in the decision to publish the results.

\section{References}

1. Nakhushev, A.M. On continual differential equations and their difference analogues. Sov. Math. Dokl. 1988, 37, 729-732.

2. Nakhushev, A.M. Positiveness of the operators of continual and discrete differentiation and integration, which are quite important in the fractional calculus and in the theory of mixed-type equations. Differ. Equ. 1998, 34, 103-112.

3. Caputo, M. Mean fractional order derivatives. Differential equations and filters. Annali dell'Univ. Ferrara Sez. VII. Sci. Mat. 1995, $41,73-84$.

4. Lorenzo, C.F.; Hartley, T.T. Variable order and distributed order fractional operators. Nonlinear Dyn. 2002, 29, 57-98. [CrossRef]

5. Sokolov, I.M.; Chechkin, A.V.; Klafter, J. Distributed-order fractional kinetics. Acta Phys. Pol. B 2004, 35, $1323-1341$.

6. Kochubei, A.N. Distributed order calculus and equations of ultraslow diffusion. J. Math. Anal. Appl. 2008, 340, 252-280. [CrossRef]

7. Caputo, M.; Fabrizio, M. The kernel of the distributed order fractional derivatives with an application to complex materials. Fractal Fracttionals 2017, 1, 13. [CrossRef]

8. Diethelm, K.; Ford, N.J. Numerical solution methods for distributed order time fractional diffusion equation. Fract. Calc. Appl. Anal. 2001, 4, 531-542.

9. Diethelm, K.; Ford, N.; Freed, A.D.; Luchko, Y. Algorithms for the fractional calculus: A selection of numerical methods. Comput. Methods Appl. Mech. Eng. 2003, 194, 743-773. [CrossRef]

10. Pskhu, A.V. On the theory of the continual and integro-differentiation operator. Differ. Equ. 2004, 40, 128-136. [CrossRef]

11. Pskhu, A.V. Partial Differential Equations of Fractional Order; Nauka Publ.: Moscow, Russia, 2005. (In Russian) 
12. Atanacković, T.M.; Oparnica, L.; Pilipović, S. On a nonlinear distributed order fractional differential equation. J. Math. Anal. Appl. 2007, 328, 590-608. [CrossRef]

13. Streletskaya, E.M.; Fedorov, V.E.; Debbouche, A. The Cauchy problem for distributed order equations in Banach spaces. Math. Notes NEFU 2018, 25, 63-72.

14. Fedorov, V.E.; Streletskaya, E.M. Initial-value problems for linear distributed-order differential equations in Banach spaces. Electron. J. Differ. Equ. 2018, 2018, 1-17.

15. Fedorov, V.E.; Abdrakhmanova, A.A. A class of initial value problems for distributed order equations with a bounded operator. In Stability, Control and Differential Games; Tarasyev, A., Maksimov, V., Filippova, T., Eds.; Springer Nature: Cham, Switzerland, 2020; pp. 251-262.

16. Fedorov, V.E.; Abdrakhmanova, A.A. Distributed order equations in Banach spaces with sectorial operators. In Transmutation Operators and Applications; Kravchenko, V.F., Sitnik, S.M., Eds.; Springer Nature: Cham, Switzerland, 2020; pp. 509-538.

17. Fedorov, V.E. On generation of an analytic in a sector resolving operators family for a distributed order equation. Zapiski POMI 2020, 489, 113-129.

18. Fedorov, V.E. Generators of analytic resolving families for distributed order equations and perturbations. Mathematics 2020, 8, 1306. [CrossRef]

19. Debbouche, A.; Fedorov, V.E. A class of fractional degenerate evolution equations with delay. Mathematics 2020, 8, 1700. [CrossRef]

20. Gerasimov, A.N. A generalization of linear laws of deformation and its application to the problems of internal friction. Prikladnaya Matematika i Mekhanika 1948, 12, 251-260. (In Russian)

21. Caputo, M.; Mainardi, F. A new dissipation model based on memory mechanism. Pure Appl. Geophys. 1971, 91, 134-147. [CrossRef]

22. Novozhenova, O.G. Life and science of Alexey Gerasimov, one of the pioneers of fractional calculus in Soviet Union. Fract. Calc. Appl. Anal. 2017, 20, 790-809. [CrossRef]

23. Bajlekova, E.G. Fractional Evolution Equations in Banach Spaces. Ph.D. Thesis, University Press Facilities, Eindhoven University of Technology, Eindhoven, The Netherlands, 2001.

24. Hille, E.; Phillips, R.S. Functional Analysis and Semi-Groups; American Mathematical Society: Providence, RI, USA, 1957.

25. Yosida, K. Functional Analysis; Springer: Berlin/Heidelberg, Germany, 1965.

26. Kato, K. Perturbation Theory for Linear Operators; Springer: Berlin/Heidelberg, Germany, 1966.

27. Da Prato, G.; Iannelli, M. Linear integro-differential equations in Banach spaces. Rend. Semin. Mat. della Univ. Padova 1980, 62, 207-219.

28. Prüss, J. Evolutionary Integral Equations and Applications; Springer: Basel, Switzerland, 1993.

29. Triebel, H. Interpolation Theory, Functional Spaces, Differential Operators; North Holland Publ.: Amsterdam, The Netherlands, 1978.

30. Arendt, W.; Batty, C.J.K.; Hieber, M.; Neubrander F. Vector-Valued Laplace Transforms and Cauchy Problems; Springer: Basel, Switzerland, 2011. 